\title{
Impact of symptoms on daily life in people at risk of rheumatoid arthritis
}

\author{
Lilian HD van Tuyl MSc PhD ${ }^{1}$ \\ Rebecca J Stack MSc PhD 2,3 \\ Maurits Sloots MSc PhD 4 \\ Lotte A van de Stadt MD PhD ${ }^{4}$ \\ Wijnanda Hoogland ${ }^{4}$ \\ Bertha Maat ${ }^{4}$ \\ Karim Raza FRCP $\mathrm{PhD}^{4,5}$ \\ Dirkjan van Schaardenburg MD PhD ${ }^{1,4}$
}

\section{Affiliations}

1 Amsterdam Rheumatology and immunology Center, VU University Medical Center, Amsterdam The Netherlands,

${ }^{2}$ Rheumatology Research Group, Institute for Inflammation and Aging, University of Birmingham, United Kingdom,

${ }^{3}$ Division of Psychology, Nottingham Trent University, United Kingdom

${ }^{4}$ Amsterdam Rheumatology and immunology Center, Reade and Academic Medical Center, Amsterdam, The Netherlands,

5 Department of Rheumatology, Sandwell and West Birmingham Hospital NHS Trust, Birmingham, UK.

\section{Corresponding author:}

Rebecca Jayne Stack

Senior Lecturer in Psychology, Nottingham Trent University, Chaucer Building, Burton St, NG14BU, United Kingdom

rebecca.stack@ntu.ac.uk 
Short title: Impact of symptoms in people at risk of rheumatoid arthritis

Word count: 1475

Key words: impact, symptoms, rheumatoid arthritis, arthralgia, psychological distress

Funding: This research has been supported in part by the FP7 HEALTH programme under the grant agreement FP7-HEALTH-F2-2012-305549 and by the Dutch Arthritis Association. 


\section{ABSTRACT}

\section{Objectives}

To explore the impact on daily life of symptoms experienced by those with systemic autoimmunity associated with rheumatoid arthritis (RA).

\section{Methods}

Fifteen people took part in focus groups to explore the impact of symptoms on daily life.

\section{Results}

Core themes included: 1) physical impact of limited hand function and mobility; 2) psychological distress experienced included a fear of symptoms progressing into RA, uncertainty about the future, feelings of despair, shame and frustration.

\section{Conclusion}

People with arthralgia at risk of developing RA experienced considerable physical and psychological impact of symptoms. Greater consideration should be given to the needs of people at risk of developing RA. 


\section{INTRODUCTION}

Rheumatoid arthritis (RA) is a chronic inflammatory joint disease that causes pain and functional impairment, which has a large impact on daily lives. Early identification and treatment improves prognosis.(van der Linden et al. 2010) To be able to identify people who will develop RA in the future, the EULAR study group for risk factors of RA recommended identification and assessment of symptoms in those with arthralgia and during the earliest phases of clinical arthritis as a research priority.(Gerlag et al. 2012) We have recently described the breadth of symptoms that persons at risk of RA might experience in the symptomatic at-risk phase.(Stack et al. 2014) Knowledge of these symptoms can facilitate the development of prediction tools and outcome measurement in arthralgia, ultimately leading to better prediction of who will develop RA.(van de Stadt et al. 2013) However, the early identification of those at risk, means that people are subject to investigations earlier, and have greater awareness of their at risk status. Research has shown the disease burden experienced by people with arthralgia is comparable to the burden experienced by people with established RA. (Kuijper et al. 2014) Furthermore, people in the earliest phases of disease may have healthcare needs that need to be addressed while being monitored (either as part of a research cohort, or in a routine healthcare setting). A recent report by Dures et al highlights the gap between the need for psychological support in early RA patients and the provision of support by rheumatology clinicians and GPs.(Dures et al. 2014a;Dures et al. 2014b) The impact of early and established RA on a patient's daily life is well researched,(Bacconnier et al. 2015;Bernatsky et al. 2010;Bury 1982;Gossec et al. 2011; Shaul 1997) but the impact and consequences of symptoms among people with increased risk of RA prior to the onset of synovial swelling have never been described by qualitative research. We describe the impact of symptoms on daily life of people at increased risk of RA.

\section{METHODS}

This report extends earlier explorations from a qualitative study on people at risk of RA and in patients with early RA to identify symptom complexes.(Stack, van Tuyl, Sloots, 
van de Stadt, Hoogland, Maat, Mallen, Tiwana, Raza, \& van Schaardenburg 2014) Ethical approval for this study was obtained from Slotervaart ethical committee.

Participants were recruited from an ongoing prospective cohort of the Jan van Breemen Research Institute | Reade in Amsterdam, The Netherlands that included 487 anti-CCP antibody positive individuals with musculoskeletal symptoms who had been referred to a rheumatologist. A total of 119 people were contacted by letter and invited to participate in one of three focus group discussions. All participants gave signed informed consent. Conduct of the discussions has been described in great detail elsewhere.[3] A semistructured interview guide was used, specifically asking: 'How do these symptoms affect your daily life? In which situations are these symptoms bothering you the most?' The interview guide was developed by LVT and RS through literature review and discussions with patient research partners (WH and BM) The group discussions took place at the Jan van Breemen Research Institute/Reade and were moderated by LVT.

Data were collected until saturation on the primary outcome (perceived symptoms) was reached and no new themes emerged. Focus group discussions lasted approximately 60 to 90 minutes and were analysed according to the inductive thematic analysis method to identify core themes in the qualitative data which explored the impact of arthralgia on daily living.(Stack, van Tuyl, Sloots, van de Stadt, Hoogland, Maat, Mallen, Tiwana, Raza, \& van Schaardenburg 2014) The transcripts where analysed by two independent researchers (LVT, MS) and one patient research partner (BM) who systematically searched for themes in the first group, forged connections between themes, then moved on to the next group. After all transcripts were analysed, the two independent researchers and the patient research partner compared their results. Differences were discussed until consensus was reached and both researchers agreed to the same set of major themes. Further data exploration created new categories, which were grouped in the themes. Coding was facilitated using NVivo software for qualitative data analysis.

\section{RESULTS}

A total of 15 individuals participated in one of three focus group discussions with two focus groups involving 4 participants and one involving 7. Participants were 11 females 
and 4 males, mean age 54 years with a mean duration from symptom onset of 12 months; two used NSAIDs plus hydroxychloroquine, five used NSAIDs only and the remaining eight did not use any drugs for arthralgia(Table 1 ).

To get a better understanding of the group of 15 participants as compared to the entire cohort of 487 participants, we compared the distribution of tender joint counts (in 53 joints), pain score (VAS scale from 0 to 100) and duration of complaints, all at cohort entry. While the focus group participants had a significantly higher tender joint count at cohort entry compared to the rest of the cohort (median 11 vs 4 ), both pain score and symptom duration were highly similar (VAS 32 vs VAS 35; 12 months vs 12 months).

This paper is focused on impact of symptoms on daily living, therefore, the themes extracted explored the physical impact and psychological impact of symptoms. Quotes supporting the themes are presented in Table 2, with physical impact quotes coded Ph1.1 to Ph1.7 and psychological impact quotes coded as Psy2.1 to Psy6.1, with 2 to 6 referring to different subthemes of psychological impact. When quotes are part of a conversation, the different participants are referred to as $\mathrm{A}, \mathrm{B}$ or $\mathrm{C}$, and the moderator as 'Mod'.

\section{Physical impact}

Participants described symptoms which led to a decrease in physical functioning and decreased participation in everyday activities. Activities such as dressing, washing, eating and household chores were impaired. Participants particularly described having problems with hand function (Ph1.1 - 1.4) and a decreased mobility (Ph1.5 - 1.6).

The impact of physical symptoms could be so severe, that participants described the psychological distress that physical symptoms had caused as reflected in quote Ph1.7.

\section{Psychological impact}

There was a general feeling of fear and uncertainty amongst the participants when describing their symptoms, as they did not know if they were at the beginning of something worse to come (i.e. RA). Several participants described the fear of future 
development of RA as the worst part of their condition (Psy2.1 - 2.3) which was closely linked to the described uncertainty about the progression of symptoms to RA in the future, influencing participants' decisions in life and making them feel limited (Psy3.1 3.5). Other described emotions included feelings of shame (Psy4.1), frustration (Psy5.1) and despair (Psy6.1).

\section{DISCUSSION}

The disruptive impact of RA on a patient's life is increasingly documented, but the impact of symptoms among people at risk of RA has never been described. Here we report on the impact of symptoms on the daily lives of anti-CCP antibody positive individuals at risk of developing RA. While the physical impact of symptoms was expected, the severity and complexity of the psychological impact of early symptoms was remarkable. Psychological distress in the form of fear, uncertainty, shame, frustration and despair in those at risk dominated the group discussions. Physicians and researchers need to realize the impact of musculoskeletal symptoms on the daily lives of anti-CCP antibody positive individuals, especially since these people may not have access to services offered to those with a confirmed diagnosis and cannot be treated with drugs with proven efficacy.

The participants that joined the discussions are part of the Reade arthralgia cohort. They are aware of their increased risk of RA and are voluntarily visiting the rheumatology clinic on a yearly basis to monitor their risk. This subgroup of people that volunteered to join a focus group discussion on perceived symptoms may differ from the larger cohort. The high tender joint count among focus group participants might imply an increased psychological impact of the symptoms in this selected group as compared to the rest of the cohort. Alternatively, comorbid conditions might have influenced the perceived physical or psychological impact of symptoms. Further exploratory investigations are needed to fully understand the needs in subgroups of people at increased risk of RA. 
Nevertheless, the described impact of musculoskeletal symptoms in anti-CCP antibody positive individuals on everyday life is substantial and should not be neglected.

It is important to understand where the psychological distress among these anti-CCP antibody positive individuals comes from in order to provide help. If making people aware of their increased risk of RA induces psychological distress, we might need to provide people with resources to deal with this information. About $35 \%$ of patients in our at-risk cohort go on to develop RA.[5] For this selected group, it is beneficial to be identified and treated as early as possible. But for the remaining $65 \%$ that never continue to develop RA, the benefit/risk ratio will be negative and the figurative 'sword of Damocles' might induce a complex spectrum of psychological distress. Like the patients that go on to develop RA, these people might benefit from psychological services and support, as well as from treatment directed at pain or other perceived symptoms.

Although focus group discussions were continued until saturation was reached, the primary purpose of the discussions was to investigate the symptoms that people with arthralgia experience, not the impact of symptoms. The impact of symptoms on daily life unexpectedly dominated the discussions. More in-depth research is needed to investigate the impact of arthralgia symptoms in other cohorts and other countries. To this end, we are currently developing a quantitative questionnaire to measure the type, severity and impact of symptoms in persons at risk of RA in order to explore commonality and prevalence of symptoms in people at risk of RA.

In conclusion, anti-CCP antibody positive individuals with musculoskeletal symptoms that have been referred to a rheumatologist, who may or may not develop RA in the future, experience substantial physical and psychological impact of symptoms on their daily life.

\section{FUNDING}

This research has been supported in part by the FP7 HEALTH programme under the grant agreement FP7-HEALTH-F2-2012-305549 and by the Dutch Arthritis Association. 


\section{CONFLICT OF INTEREST STATEMENT}

The authors have no competing interests regarding this work 
Table 1: Participants in one of three focus group discussions

\begin{tabular}{|c|c|c|c|c|}
\hline Participant & $\begin{array}{l}\text { Tender joint } \\
\text { count } \\
(0 \text { to } 53 \text { ) }\end{array}$ & $\begin{array}{l}\text { Pain } \\
\text { (VAS scale 0- } \\
100)\end{array}$ & $\begin{array}{l}\text { Duration of } \\
\text { complaints before } \\
\text { cohort entry }\end{array}$ & Medication usage \\
\hline 1 & 0 & 50 & 6 & No \\
\hline 2 & 0 & 15 & 25 & No \\
\hline 3 & 2 & 0 & 12 & No \\
\hline 4 & 2 & 15 & 9 & HCQ and NSAIDs \\
\hline 5 & 6 & 0 & 12 & No \\
\hline 6 & 6 & 71 & 24 & No \\
\hline 7 & 8 & 40 & 12 & NSAIDs \\
\hline 8 & 8 & 25 & 5 & HCQ and NSAIDs \\
\hline 9 & 6 & 49 & 22 & No \\
\hline 10 & 11 & 40 & 12 & NSAIDs \\
\hline 11 & 8 & 24 & 12 & NSAIDs \\
\hline 12 & 0 & 40 & 4 & NSAIDs \\
\hline 13 & 1 & 55 & 8 & No \\
\hline 14 & 0 & 54 & 8 & NSAIDs \\
\hline 15 & 0 & 3 & 18 & No \\
\hline
\end{tabular}

Table 2: Quotes supporting the themes regarding the impact of participants' symptoms

\footnotetext{
Physical impact

Ph1.1 P11: 'In the hands. Especially when closing buttons, I can't close buttons.'

P12: 'I just leave them closed [red: buttons], and just pull it over my head.'

Ph1.2 P2: 'Like cleaning potatoes, I don't do that anymore. Because I notice...and that frustrates me, so I buy clean potatoes'.
}

Ph1.3 P3: 'Well, opening a jar, opening the water faucet, taking something, ...' 
Ph1.4 P12: 'I played the piano a lot and I can't do that anymore, not as fast as I used to, especially not when I am having an attack, so those kind of things...'

Ph1.5 P5: 'That you can't make certain movements. One day, I wanted to rise from my chair and I just fell backwards, I couldn't walk.'

Ph1.6 P6: 'The mobility. It can just surprise you, that you suddenly can't do anything. With cooking, everything is clumsy.'

P5: 'Yes, and peeling potatoes'

Ph1.7 P7: 'Just opening a bottle of Coke when you have friends over, then you have to ask someone to open it for you. That makes me very sad at my age' [red: Lady is 46]

\section{Psychological impact}

Fear of future development of RA

Psy2.1 P12: 'That is what bothers me the most. That idea of: "Oh now I get it [referring to $\mathrm{RA}]$, now it starts".'

P13: 'Because it never lasts long, I know it will always subside. I think what troubles me the most is the hope it doesn't get worse. If it remains like this, I can live with it.'

Psy2.2 P9: 'I was referred to the rheumatologist, so yes, that fear that I could now be entering a phase of becoming a patient. That's really uncomfortable.'

Psy2.3 P11: 'Uncertainty about it becoming worse or not; if I knew this level could be stabilized, that this would be it, I would sign up for that immediately.'

\section{Uncertainty}

Psy3.1 P14: 'I always said I would never marry again, but last year I did. I am a wedding officer, so I feel warmly about marriage, but I thought no, I will not do that again. I have three kids, and no. And then I thought, yes, but this is a guy that will, if I end up in a wheelchair, I hope not but if I do, I am certain that he will push me around.' Mod: 'And did that play a role?'

P14: 'that has certainly played a role in my decision.'

P15: 'that is the certainty that gives you some air.'

P14: 'If I didn't have this [red: arthralgia] I might not have married him, we would have just lived together.'

Psy3.2 P7: 'Well, I have been honest with my boss. I told her, one moment it's worse than the other moment. My colleagues know it as well. But I can imagine, I have that uncertainty as well, especially now and for the future, since the new generation is 
coming. If they can hire a young person, without any complaints, then I'm the first to go.'

Psy3.3 P4: 'I have a problem with that, that you think 'what will happen'? I'm 59, alone for 4 years, and then you start thinking...'

P5: 'who will take care of me?'

P7: 'Yes, who takes care of me, that's what I say. And then you think, my boss is trying to push me out as well, partly because of my symptoms, so...'

P4: 'uncertain future.'

Psy3.4 P12: 'that is what is bothering me about these symptoms, every time you think, is it because of this?[red: development of RA] That is annoying, that uncertainty is very annoying for me.'

P13: 'I recognise that, because two weeks ago I got a sudden pain in my wrist and then you start to think is it because of this, or is it just because I hit it? I really don't know.'

P14: 'It's the fear as well.'

P13: 'It disappeared again so I was relieved, that it might not be those symptoms that are related to RA. Because that's with everything you feel now, or when you drop something. You think "Oh no, now it starts", yes I recognize that.'

Psy3.5 P10: 'If I know what it is, then something might be done, maybe, or at least... I really want to know what it is.'

Shame

Psy4.1 P7: 'You feel ashamed too. I don't dare to drink anything in large company; yes I bring a bottle of water, that can't do much harm[red: shame of dropping her drink]. And that annoys me. It is quite a burden.'

P6: 'We are all rather young to be having such complaints, so that's frustrating, and that makes me sad. I find that hard to cope with. Sometimes when I get out of bed in the morning and I am slower than usual getting ready for work, and when I enter the office reasonably on time they always say something like 'good afternoon!' I told them during a meeting recently that these remarks are hurtful to me. I understand it's a joke, but it is hurtful. And I think that is your feeling as well, not even the shame, but because you are afraid people will say something about it, and that's annoying for you.'

\section{Frustration}




\begin{abstract}
Psy5.1 P6: 'A big part of it is frustration, but I also notice it makes me very sad, because I think we are still so young. These are symptoms you would expect in much older people.

\section{$\underline{\text { Despair }}$}

Psy6.1 P7: 'Yes, just painful, swollen, really hurting. I couldn't do a thing. And then you come there and that guy [rheumatology intern on duty] says it's overburdening or an acute form of arthritis, but that's it. You get a box with drugs and it should get better within 24 hours. They don't check the blood, nothing, and right after that you get another attack. Then I think, in God's name where do I go with this kind of attack? Because you can't do a thing. My husband was shocked, because I have relatively thin wrists, but they became twice the size and my hands too. You can't do a thing, it's so painful. The tears are running down your face, you walk, can't sit still, because you don't know how to put your hands. You walk around the room, not knowing what to do, you sit down again. It drove me crazy, I was bordering on a depression, if this takes any longer I will just throw myself off ... I will just quit. I thought if this is what awaits me, than it's over, I don't need that.'
\end{abstract}

\title{
References
}

Bacconnier, L., Rincheval, N., Flipo, R.M., Goupille, P., Daures, J.P., Boulenger, J.P., \& Combe, B. 2015. Psychological distress over time in early rheumatoid arthritis: results from a longitudinal study in an early arthritis cohort. Rheumatology.(Oxford), 54, (3) 520-527 available from: PM:25224416

Bernatsky, S., Feldman, D., De, C.M., Haggerty, J., Tousignant, P., Legare, J., Zummer, M., Meagher, T., Mill, C., Roper, M., \& Lee, J. 2010. Optimal care for rheumatoid arthritis: a focus group study. Clin.Rheumatol., 29, (6) 645-657 available from: PM:20127397

Bury, M. 1982. Chronic illness as biographical disruption. Sociol.Health IIIn., 4, (2) 167-182 available from: PM:10260456 
Dures, E., Almeida, C., Caesley, J., Peterson, A., Ambler, N., Morris, M., Pollock, J., \& Hewlett, S. 2014a. A survey of psychological support provision for people with inflammatory arthritis in secondary care in England. Musculoskeletal.Care, 12, (3) 173-181 available from: PM:24753071

Dures, E., Almeida, C., Caesley, J., Peterson, A., Ambler, N., Morris, M., Pollock, J., \& Hewlett, S. 2014b. Patient preferences for psychological support in inflammatory arthritis: a multicentre survey. Ann Rheum Dis available from: PM:25261572

Gerlag, D.M., Raza, K., van Baarsen, L.G., Brouwer, E., Buckley, C.D., Burmester, G.R., Gabay, C., Catrina, A.I., Cope, A.P., Cornelis, F., Dahlqvist, S.R., Emery, P., Eyre, S., Finckh, A., Gay, S., Hazes, J.M., van der Helm-van Mil, Huizinga, T.W., Klareskog, L., Kvien, T.K., Lewis, C., Machold, K.P., Ronnelid, J., van, S.D., Schett, G., Smolen, J.S., Thomas, S., Worthington, J., \& Tak, P.P. 2012. EULAR recommendations for terminology and research in individuals at risk of rheumatoid arthritis: report from the Study Group for Risk Factors for Rheumatoid Arthritis. Ann Rheum Dis, 71, (5) 638-641 available from: PM:22387728

Gossec, L., Paternotte, S., Aanerud, G.J., Balanescu, A., Boumpas, D.T., Carmona, L., de, W.M., Dijkmans, B.A., Dougados, M., Englbrecht, M., Gogus, F., Heiberg, T., Hernandez, C., Kirwan, J.R., Mola, E.M., Cerinic, M.M., Otsa, K., Schett, G., Scholte-Voshaar, M., Sokka, T., von, K.G., Wells, G.A., \& Kvien, T.K. 2011. Finalisation and validation of the rheumatoid arthritis impact of disease score, a patient-derived composite measure of impact of rheumatoid arthritis: a EULAR initiative. Ann Rheum Dis, 70, (6) 935-942 available from: PM:21540201

Kuijper, T.M., Luime, J.J., Alves, C., Barendregt, P.J., van, Z.J., Bindels, P.J., \& Hazes, J.M. 2014. Quality of life and health care use in patients with arthralgias without synovitis compared with patients diagnosed with early rheumatoid arthritis: data from an early arthritis cohort. Arthritis Care Res.(Hoboken.), 66, (3) 379-386 available from: PM:23982959

Shaul, M.P. 1997. Transitions in chronic illness: rheumatoid arthritis in women. Rehabil.Nurs., 22, (4) 199-205 available from: PM:9275811

Stack, R.J., van Tuyl, L.H., Sloots, M., van de Stadt, L.A., Hoogland, W., Maat, B., Mallen, C.D., Tiwana, R., Raza, K., \& van Schaardenburg, D. 2014. Symptom complexes in patients with seropositive arthralgia and in patients newly diagnosed with rheumatoid arthritis: a qualitative exploration of symptom development. Rheumatology (Oxford), 53, (9) 1646-1653 available from: PM:24729397

van de Stadt, L.A., Witte, B.I., Bos, W.H., \& van Schaardenburg, D. 2013. A prediction rule for the development of arthritis in seropositive arthralgia patients. Ann Rheum Dis, 72, (12) 2021-2027 available from: PM:23178208

van der Linden, M.P., le Cessie S., Raza, K., van der Woude, D., Knevel, R., Huizinga, T.W., \& van der Helm-van Mil AH 2010. Long-term impact of delay in assessment of early arthritis patients. Arthritis Rheum., 62, (12) 3537-3546 available from: PM:20722031 